\title{
Real-space renormalization group flow in quantum impurity systems: local moment formation and the Kondo screening cloud
}

\author{
Andrew K. Mitchell, Michael Becker and Ralf Bulla ${ }^{1}$ \\ ${ }^{1}$ Institute for Theoretical Physics, University of Cologne, 50937 Cologne, Germany
}

(Dated: November 30, 2018)

\begin{abstract}
The existence of a length-scale $\xi_{K} \sim 1 / T_{K}$ (with $T_{K}$ the Kondo temperature) has long been predicted in quantum impurity systems. At low temperatures $T \ll T_{K}$, the standard interpretation is that a spin- $\frac{1}{2}$ impurity is screened by a surrounding 'Kondo cloud' of spatial extent $\xi_{K}$. We argue that renormalization group (RG) flow between any two fixed points (FPs) results in a characteristic length-scale, observed in real-space as a crossover between physical behaviour typical of each FP. In the simplest example of the Anderson impurity model, three FPs arise; and we show that 'free orbital', 'local moment' and 'strong coupling' regions of space can be identified at zero temperature. These regions are separated by two crossover length-scales $\xi_{\mathrm{LM}}$ and $\xi_{K}$, with the latter diverging as the Kondo effect is destroyed on increasing temperature through $T_{K}$. One implication is that moment formation occurs inside the 'Kondo cloud', while the screening process itself occurs on flowing to the strong coupling FP at distances $\sim \xi_{K}$. Generic aspects of the real-space physics are exemplified by the two-channel Kondo model, where $\xi_{K}$ now separates 'local moment' and 'overscreening' clouds.
\end{abstract}

PACS numbers: 72.15.Qm, 73.63.Kv, 75.20.Hr

\section{INTRODUCTION}

Most fundamental aspects of the Kondo effect are by now very well understood, ${ }^{1}$ with various detailed theoretical predictions having been confirmed directly by experiments on impurity systems or quantum dot devices. ${ }^{1,2}$ Key insights into the underlying physics have been provided by the renormalization group (RG) concept, where progressive reduction of the temperature or energy scale results in RG flow between 'fixed points' (FPs) that can be easily identified for a given model. In particular, the low-temperature/energy physics is governed by RG flow between two fundamental FPs, with universality arising in terms of the crossover energy scale.

The Anderson impurity model (AIM) captures many generic aspects of quantum impurity physics. Three FPs (and hence two crossover energy scales) arise: charge fluctuations on the impurity or quantum dot dominate at the 'free orbital' (FO) FP, which describes the high-energy physics. But due to strong impurity electron correlations, a spin- $\frac{1}{2}$ 'local moment' (LM) forms below $T \sim T_{\mathrm{LM}}$ (typically a high-energy scale). RG flow from this LM FP to the 'strong coupling' (SC) FP occurs on a much lower energy scale $T_{K}$, and determines the universal low-energy behaviour. The SC FP itself describes the ground state in which the incipient moment associated with the LM FP is dynamically screened by conduction electrons, which together form a many-body 'Kondo singlet' state. ${ }^{1}$

Surprisingly however, the underlying physics in realspace is still somewhat controversial. Various theoretical studies ${ }^{3-14}$ have examined real-space behaviour in a number of impurity models. While they do appear to have established the existence of a Kondo length-scale, $\xi_{K} \equiv \hbar v_{F} / k_{B} T_{K}$ (with $v_{F}$ the Fermi velocity), the nature of the two spatial regions separated by it remains unclear. Certain quantities have been studied in the universal scaling regime, ${ }^{3-14}$ but the full and exact evolution of real-space quantities - and a satisfactory unifying in- terpretation of the results - has not yet been provided. The prevailing view ${ }^{15}$ is that at low temperatures $T \ll T_{K}$, a spin- $\frac{1}{2}$ impurity forms a singlet by binding to a surrounding 'Kondo cloud' of spatial extent $\xi_{K}$. This has an intuitive appeal from the perspective of Fermi liquid theory, where one imagines that the impurity is 'invisible' to conduction electrons outside the screening cloud. ${ }^{15}$ But no clear evidence in support of the screening cloud scenario has so far emerged. Very recently, refinement of scanning tunneling spectroscopy techniques reported in Ref. 16 has shown for the first time that Kondo signatures in the local density of states (LDOS) appear away from an impurity. Experimental verification of the elusive Kondo length-scale - and resolution of the realspace debate - may now finally be within reach.

Motivated in part by these developments, we consider here the wider question of how RG flow manifests in real-space. While necessarily perturbative, a simple intuitive picture is suggested by well-known scaling arguments applied directly to real-space, ${ }^{17}$ where notions of RG and universality again arise. One might anticipate then that this universality could show up in certain real-space quantities. Indeed, some concrete evidence

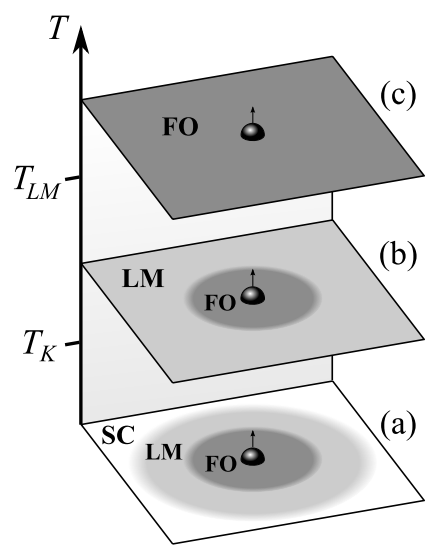

Figure 1: Three physical regions arise at $T=0$ in the Anderson impurity model, corresponding to the FO, LM and SC FPs (a). The crossover length-scale $\xi_{K}$ associated with flow to the SC FP diverges as the Kondo effect is destroyed on increasing temperature through $T_{K}(\mathrm{~b})$, while $\xi_{\mathrm{LM}}$ diverges for $T \gg$ $T_{\mathrm{LM}}(\mathrm{c})$. The spin- $\frac{1}{2}$ moment forming at $\xi_{\mathrm{LM}}$ is screened on flowing to the SC FP at $\xi_{K}$. 
to support this general expectation has been provided theoretically ${ }^{3-14}$ if not experimentally.

Encouraged by these results, we show in this paper that motion away from an impurity corresponds to RG flow. In fact, we demonstrate explicitly and exactly that signatures of the entire RG flow between all FPs (whether universal or non-universal) must appear fully in real-space. Just as flow between a pair of FPs results in a characteristic energy scale, we show that one can similarly identify the corresponding length-scale, apparent in real-space as a crossover between physical behaviour typical of each FP. In particular, this implies that length-scales can be attributed both to moment formation and moment compensation. In real impurity or quantum dot systems, the moment screened by the Kondo effect at low temperatures does not reside on the impurity or dot itself, but is an extended object which develops in real-space due to electron correlations.

Further insight is gained by considering the effect on the real-space physics of increasing temperature. RG flow is cut off at an energy scale $\sim T$; likewise RG flow is arrested on the thermal length-scale $\xi_{T} \propto 1 / T$. Consequently, clouds associated with low-energy FPs reached at larger distances 'evaporate' successively on increasing temperature. Our refined real-space Kondo screening scenario is summarized schematically in Fig. 1.

\section{ANDERSON IMPURITY MODEL IN REAL-SPACE}

For simplicity and concreteness, we consider first a semi-infinite tight-binding chain, $H_{\text {host }}=t \sum_{\sigma} \sum_{i=0}^{\infty}\left(c_{i, \sigma}^{\dagger} c_{i+1, \sigma}+\right.$ H.c $)$, with a single Anderson impurity tunnel-coupled to one end:

$$
H_{\text {And }}=H_{\text {host }}+\epsilon \hat{n}_{d}+U \hat{n}_{d, \uparrow} \hat{n}_{d, \downarrow}+V \sum_{\sigma}\left(d_{\sigma}^{\dagger} c_{0, \sigma}+\text { H.c }\right),
$$

where $\hat{n}_{d}=\sum_{\sigma} \hat{n}_{d, \sigma} \equiv \sum_{\sigma} d_{\sigma}^{\dagger} d_{\sigma}$ is the total number operator for the impurity, and the hybridization strength is $\Gamma=\pi \rho V^{2}$ (with $\rho=1 /(\pi t)$ the Fermi level density of states and $2 t \equiv 1$ the half-bandwidth). As in previous theoretical work, ${ }^{3-6}$ we focus here on the 'excess' charge density due to the impurity, obtained via

$$
\Delta n(r, T)=-\frac{2}{\pi} \operatorname{Im} \int_{-\infty}^{+\infty} d \omega f(\omega, T) \Delta G_{r, \sigma}(\omega, T),
$$

with $f(\omega, T)=[1+\exp (\omega / T)]^{-1}$ the Fermi function and $\Delta G_{r, \sigma}(\omega, T) \equiv G_{r, \sigma}(\omega, T)-G_{r}^{0}(\omega)$ the difference in the site- $r$ Green function with and without the impurity.

The free conduction chain is initially at half-filling, and the corresponding no-impurity propagators $G_{r}^{0}(\omega)$ are simple objects, describing non-interacting electrons. By contrast, the behaviour of the full Green function $G_{r, \sigma}(\omega, T) \equiv\left\langle\left\langle c_{r, \sigma} ; c_{r, \sigma}^{\dagger}\right\rangle\right\rangle_{\omega, T}$ is deeply non-trivial due to electron correlations on the impurity which are ultimately responsible for local moment formation and thence the Kondo effect. But these correlations are lo$c a l$, and so the subtle real-space behaviour of $G_{r, \sigma}(\omega, T)$
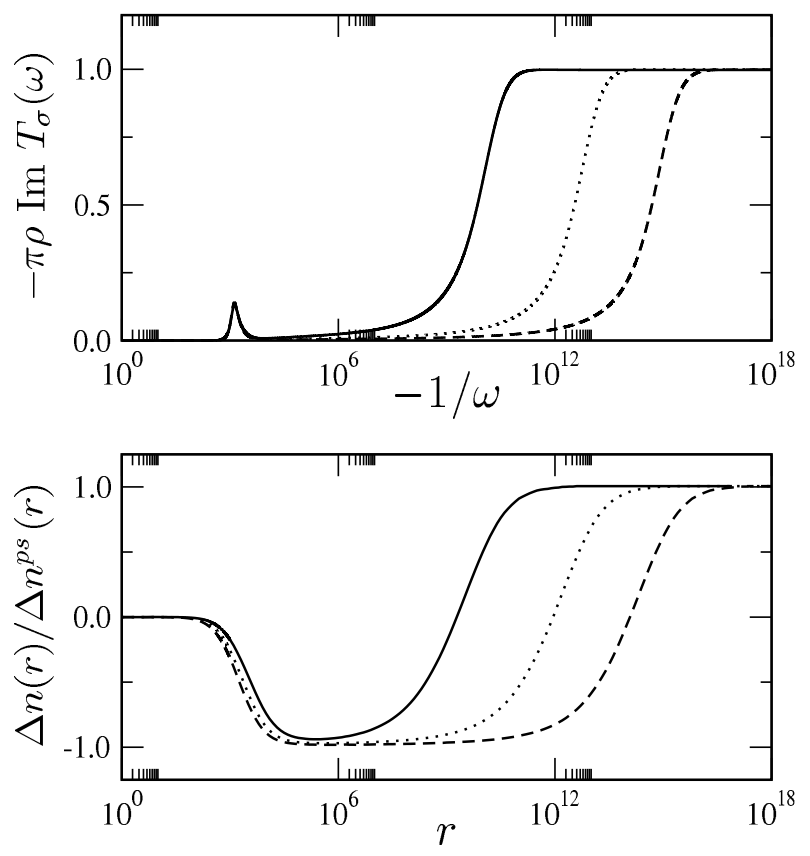

Figure 2: Comparison of the spectrum vs inverse frequency (upper panel) and normalized excess charge density vs distance (lower panel) for the Anderson model at $T=0$. Plotted for common $10^{3} \mathrm{~V}=3$ and $10^{3} \epsilon=-0.8$, varying $10^{3} \mathrm{U}=1$, 1.125 and 1.25 (solid, dotted and dashed lines), chosen to give exaggerated energy- and length-scale separations for clarity.

and $\Delta n(r, T)$ can be determined from purely local impurity quantities. This is most clearly seen using equations of motion, ${ }^{6}$ whence for the simple geometry considered here, one readily obtains

$$
\Delta G_{r, \sigma}(\omega, T)=\left[\frac{1}{t} X(\omega, r)\right]^{2} T_{\sigma}(\omega, T) .
$$

All the real-space information about the lattice is contained in the simple function $X(\omega, r)=\left[t G_{0}^{0}(\omega)\right]^{r+1}$, where $G_{0}^{0}(\omega)=\left(\omega-\sqrt{\omega^{2}-4 t^{2}}\right) / 2 t^{2}$ is the free $r=0$ Green function. The spatial dependence of $\Delta G_{r, \sigma}(\omega, T)$ thus enters only through the power of $G_{0}^{0}(\omega)$, reflecting physically the fact that electrons must hop $(r+1)$ times to get to the impurity. $T_{\sigma}(\omega, T)=V^{2} G_{d, \sigma}(\omega, T)$ is the usual scattering t-matrix, with $G_{d, \sigma}(\omega, T) \equiv\left\langle\left\langle d_{\sigma} ; d_{\sigma}^{\dagger}\right\rangle\right\rangle_{\omega, T}$ the impurity Green function. In the trivial non-interacting limit $(U=0)$, the impurity merely gives rise to potential scattering, and the familiar Friedel density oscillations are expected. ${ }^{18}$ In this case $G_{d, \sigma}^{p s}(\omega)=[\omega-$ $\left.\epsilon-V^{2} G_{0}^{0}(\omega)\right]^{-1}$ independent of temperature. Far from the impurity, Eqs. 2 and 3 reduce to $\Delta n^{p s}(r, T)=$ $-\frac{2}{\pi} \operatorname{Im} f(2 r, T) \cdot(-1)^{r} C$, with $f(r, T)=-i \pi T / \sinh (\pi r T)$ the Fourier transform of the Fermi function, and where $C=2 \epsilon \Gamma /\left(\epsilon^{2}+\Gamma^{2}\right)=\sin (2 \delta)$ depends purely on the Fermi liquid phase shift $\delta$. At low-temperatures $T \ll 1 / r$, one obtains asymptotically $\Delta n^{p s}(r, T)=$ $\frac{C}{\pi}(-1)^{r} / r$, while at higher temperatures, density oscillations are exponentially suppressed via $\Delta n^{p s}(r, T)=$ $4 C T(-1)^{r} \exp \left(-2 \pi r / \xi_{T}\right)$, with the thermal length-scale $\xi_{T}=1 / T$ so defined, and appearing naturally. 

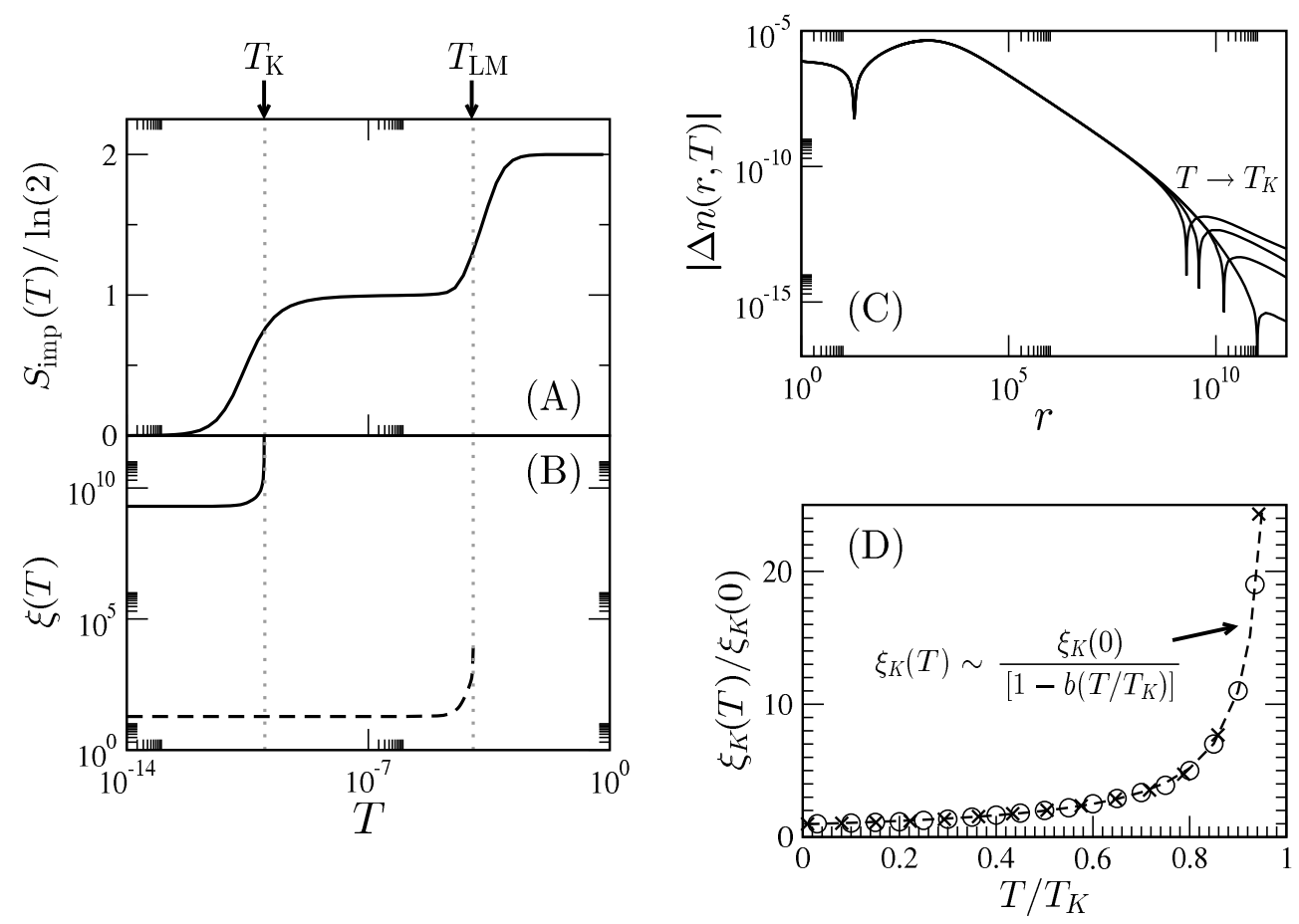

Figure 3: Effect of temperature on the Anderson model $\left(10^{3} \mathrm{~V}=3,10^{3} \epsilon=-0.8,10^{3} \mathrm{U}=1\right)$. Panels (A) and (B): Comparison of impurity entropy with length-scales $\xi_{K}$ (solid lines) and $\xi_{\mathrm{LM}}$ (dashed) as they evolve with $T$. (C): $|\Delta n(r, T)|$ vs $r$ for $T / T_{K}=0.1,0.5,0.75$ and 0.85 . (D): $\xi_{K}(T) / \xi_{K}(0)$ vs $T / T_{K}$ for the same system (circles), and scaling collapse of the divergence to common universal curve for $V=0.05, \epsilon=-0.1, U=0.3$ (crosses). Dashed line is a fit to the data.

The situation of interest is of course the $U>0$ interacting AIM, where $G_{r, \sigma}(\omega, T)$ and hence $T_{\sigma}(\omega, T)$ now contain information about the Kondo effect. In this work we employ the numerical renormalization group (NRG) to obtain accurately $T_{\sigma}(\omega, T) \cdot{ }^{19}$ Provided $T \ll T_{K}$, signatures of Kondo physics and RG flow are naturally expected in the Green function or LDOS for any site in real-space (see Eq. 3), and this has been recently confirmed experimentally. ${ }^{16}$ But the factorized form of Eq. 3 implies that such measurements cannot reveal a Kondo length-scale, $\xi_{K}$ (there can be no universality in terms of $\left.r / \xi_{K}\right)$.

The key point is that the real-space charge densities are obtained from a simple integral transformation of the frequency-resolved t-matrix by Eqs. 2 and 3. Indeed, far from the impurity this transformation reduces to a Fourier transformation, whose well-defined inverse guarantees the complete conservation of information for the process $T_{\sigma}(\omega, T) \leftrightarrow \Delta n(r, T)$. In consequence, the full $R G$ structure of the underlying quantum impurity problem (as manifest in the t-matrix or LDOS) is wholly reproduced in the spatial variation of $\Delta n(r, T)$.

This fact is shown at zero-temperature in Fig. 2, where the energy-dependence of the spectrum $-\pi \rho \operatorname{Im} T_{\sigma}(\omega)$ is compared with the space-dependence of the densities $\Delta n(r) / \Delta n^{p s}(r)$, normalized by the pure potential scattering contribution at long distances (with $C=\sin (2 \delta)$ and $\delta$ now the phase shift of the interacting problem). The representative systems plotted in Fig. 2 have similar energy scales $T_{\mathrm{LM}} \sim \mathcal{O}(U)$, but widely differing Kondo scales $T_{K}$. The densities shown in the lower panel exhibit clearly RG flow between FPs, now as a function of distance: a non-universal crossover length-scale $\xi_{\mathrm{LM}}$ characterizes moment formation on flowing from FO to LM FPs, while $\xi_{K}$ sets the universal length-scale for Kondo screening of this moment on flowing to the SC FP.

Since the SC FP can be thought of as a free conduction band with one site removed, a $\pi / 2$ phase shift results. ${ }^{1}$ As shown in Ref. 4 for the Kondo model, this is responsible for a sign-change in the Friedel oscillations. Indeed, this provides a natural explanation of the limiting universal values $\Delta n(r) / \Delta n^{p s}(r)=-1$ and +1 for $r \ll \xi_{K}\left(\gg \xi_{\mathrm{LM}}\right)$ and $r \gg \xi_{K}$; and the resulting minimum in $|\Delta n(r)|$ allows direct identification of the Kondo length-scale. However, we point out that the FO FP can similarly be thought of as a free conduction band with an additional potential scatterer. The resulting phase shift $\delta$ also results in a minimum of $|\Delta n(r)|$ at $r=\xi_{\mathrm{LM}}$. Both length-scales of the Anderson model can be simply identified in this way, and we find $\xi_{K} \sim 1 / T_{K}$ and $\xi_{\mathrm{LM}} \sim 1 / T_{\mathrm{LM}}$; consistent with expectations based on simple scaling grounds. In particular, $\xi_{K}$ grows as the impurity-host coupling decreases. ${ }^{1}$ Indeed, Kondo screening is totally absent in the limit of an uncoupled impurity $V \rightarrow 0$. Here local moment physics persists and $\xi_{K}$ diverges. This implies directly that the spatial region $r \ll \xi_{K}\left(\gg \xi_{\mathrm{LM}}\right)$ is described by the LM FP, and cannot be as such a 'screening cloud'.

One naturally expects the RG structure of the problem to be manifest also in the temperature-dependence 
of physical quantities. This is strikingly apparent in thermodynamics such as the entropy, ${ }^{19}$ whose impurity contribution flows from $S_{\mathrm{imp}}(T)=\ln (4)$ at the FO FP to $\ln (2)$ at the LM FP, and finally $S_{\mathrm{imp}}=0$ at the SC FP, symptomatic of complete Kondo screening; and as shown in Fig. 3(A). The corresponding effect of temperature on the real-space physics is demonstrated in panels $(\mathrm{B})-(\mathrm{D})$. Two clear dips in $|\Delta n(r, T)|$ are seen at each temperature in panel $(\mathrm{C})$, allowing thereby immediate identification of $\xi_{\mathrm{LM}}(T)$ and $\xi_{K}(T)$. As the temperature is increased through $T_{K}$, we find that $\xi_{K}(T)$ moves out to larger distances (while $\xi_{\mathrm{LM}}(T)$ remains unaffected). On destroying the Kondo effect by increasing temperature, one would expect a 'screening cloud' per se to collapse; but understanding $\xi_{K}$ instead as the length-scale for RG flow from LM to SC FPs, the divergence of $\xi_{K}(T)$ indicates simply the spatial persistence of local moment physics. Temperature cuts off RG flow to the SC FP, and so there can be no crossover Kondo length-scale to an SC region of space for $T_{K} \ll T \ll T_{\mathrm{LM}}$. The LM FP, describing an unscreened impurity, then pertains for $r \gg \xi_{\mathrm{LM}}$.

The characteristic divergence of $\xi_{K}(T)$ exhibits universal scaling in terms of $T / T_{K}$, as shown in panel (D). This behaviour is found to be described by $\xi_{K}(T) \sim$ $\xi_{K}(0) /\left[1-b\left(T / T_{K}\right)\right]$ (dashed line), with $b=\mathcal{O}(1)$; and as such the diverging length-scale corresponds roughly to the vanishing energy scale $\left(T_{K}-T\right)$. The full evolution of $\xi_{K}(T)$ and $\xi_{\mathrm{LM}}(T)$ is shown in Fig. 3(B). The free moment associated with the LM FP is destroyed by charge fluctuations at the FO FP when $T \gg T_{\mathrm{LM}} \cdot \xi_{\mathrm{LM}}$ thus diverges on warming through $T_{\mathrm{LM}}$. The real-space behaviour in the three distinct temperature regimes is illustrated pictorially in Fig. 1.

\section{MULTI-CHANNEL KONDO MODEL}

To emphasize the generality of the above results, we turn now to the $N$-channel Kondo model, ${ }^{20,21}$ given by

$$
H_{K}^{N}=\sum_{i=1}^{N}\left(H_{\mathrm{host}}^{i}+J_{i} \hat{\mathbf{S}} \cdot \hat{\mathbf{s}}_{i}\right)
$$

describing a single spin- $\frac{1}{2}$ impurity $\hat{\mathbf{S}}$ exchange-coupled to the conduction electron spin-density $\hat{\mathbf{s}}_{i}$ of channel $i$ at the impurity. As before we consider a linear chain geometry, but with potential scattering ${ }^{1}$ in each channel now included explicitly since the impurity is now strictly singly-occupied: $H_{\text {host }}^{i}=\sum_{\sigma}\left\{K c_{i, 0, \sigma}^{\dagger} c_{i, 0, \sigma}+\right.$ $\sum_{r=0}^{\infty} t\left(c_{i, r, \sigma}^{\dagger} c_{i, r+1, \sigma}+\right.$ H.c $\left.)\right\}$. Straightforward application of the equations of motion yield directly an analogue of Eq. 3 for each conduction channel $i$ :

$$
\Delta G_{i, r, \sigma}(\omega, T)=\Delta G_{r}^{p s}(\omega)+\left[\frac{1}{t} \tilde{X}(\omega, r)\right]^{2} T_{i \sigma}(\omega, T),
$$

where $\Delta G_{r}^{p s}(\omega)$ is the trivial potential scattering contribution in the $J_{i}=0$ case, $\tilde{X}(\omega, r)=X(\omega, r) /[1-$ $\left.K G_{0}^{0}(\omega)\right]$ contains the lattice information, and $T_{i \sigma}(\omega, T)$ are the t-matrices of the multi-channel Kondo model
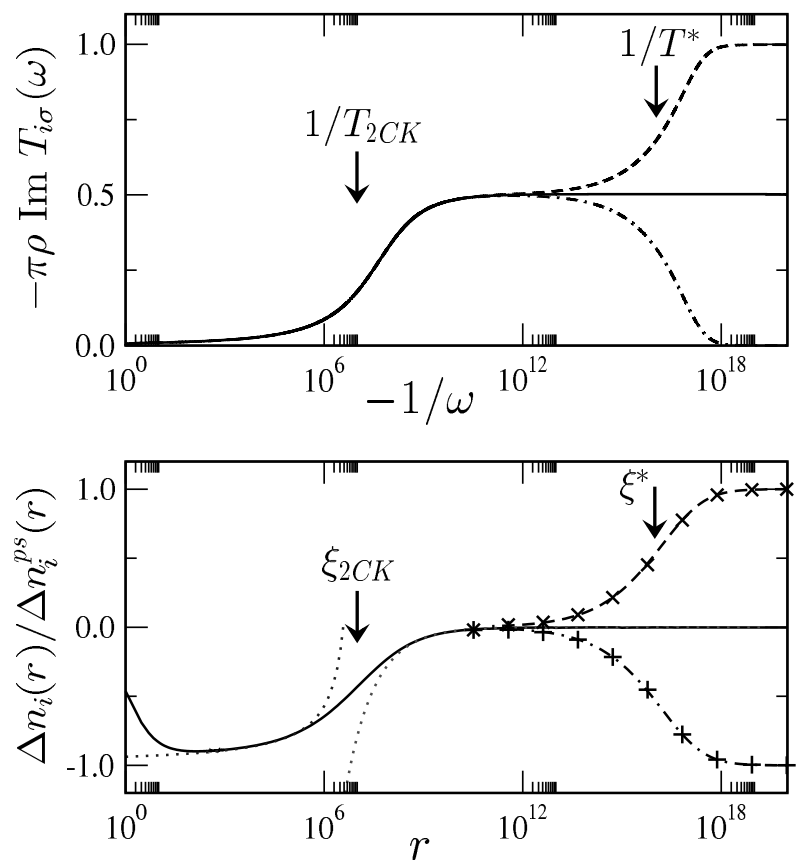

Figure 4: $T=0$ spectrum vs inverse frequency (upper panel) and normalized density vs distance (lower panel) for the $2 \mathrm{CK}$ model with $\left(J_{1}+J_{2}\right)=0.2$. Symmetric case $J_{1}=J_{2}$ shown as solid lines, while results for channel 1(2) shown as the dashed(dot-dashed) lines in the asymmetric case $\left(J_{1}-J_{2}\right)=10^{-7}$. Common asymptotic behaviour for $r \ll \xi_{2 C K}$ is $\Delta n_{i}(r) / \Delta n_{i}^{p s}(r) \sim c / \ln ^{2}\left(r / \xi_{2 C K}\right)-1$ while $\sim\left(r / \xi_{2 C K}\right)^{-1 / 2}$ behaviour is observed for $r \gg \xi_{2 C K}\left(\ll \xi^{*}\right)$. In the asymmetric case, excellent agreement is seen with the exact result in the vicinity of the FL crossover, Eq. 6 (points).

(again obtained here using NRG). Friedel oscillations in each channel arise when $K \neq 0$ due to potential scattering [with $C=\sin (2 \delta)=-2 K t /\left(K^{2}+t^{2}\right)$ ]. But non-trivial many-body effects associated with the multichannel Kondo model also appear in the real-space charge densities $\Delta n_{i}(r, T)$ for $J_{i} \neq 0$, as now shown for the $N=2$ two-channel Kondo (2CK) model. ${ }^{20,21}$

In the channel-symmetric case $J_{1}=J_{2}>0, \mathrm{RG}$ flow between two FPs determines the underlying physics. The LM FP describes the behaviour at high energies/temperatures, where the impurity spin is essentially free. But intrinsic frustration arising from symmetric coupling to two conduction channels drives the system to the 2CK FP below $T_{2 C K}$, which describes a non-Fermi liquid (NFL) ground state characterized by 'overscreening' of the impurity spin. ${ }^{20,21}$ This RG flow is of course clearly manifest in the behaviour of the t-matrix for each channel, $T_{i \sigma}(\omega)$ (equivalent by symmetry), and so we expect to observe the same flow in real-space.

An analog to the single-channel 'Kondo screening cloud' has been suggested for the symmetric 2CK model: ${ }^{14}$ an 'overscreening' cloud of spatial extent $\xi_{2 C K} \sim 1 / T_{2 C K}$ surrounding a partially quenched impurity. But since the ground state is a NFL, what lies beyond $\xi_{2 C K}$ ? Within the present RG description, the 


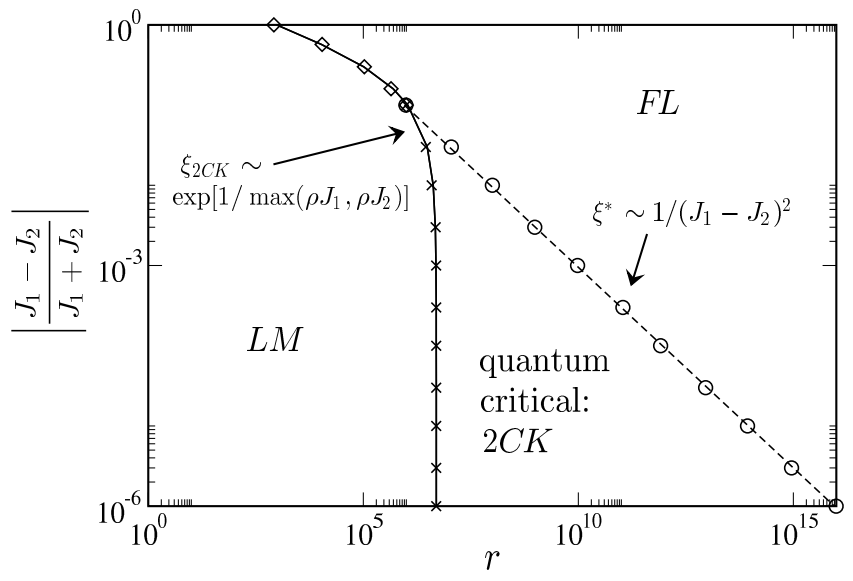

Figure 5: $T=0$ phase diagram for the 2CK model, showing LM, critical 2CK and FL regions of space. Direct crossover from LM to FL occurs for large asymmetry (diamond points), but a quantum critical region of space appears for smaller asymmetry. Two distinct scales are observed in this regime: $\xi_{2 C K}$ (crosses) and $\xi^{*}$ (circles). Solid line is fit to expected behaviour $\xi_{2 C K} \equiv 1 / T_{2 C K}$ while dashed line is $\xi^{*} \equiv 1 / T^{*}$.

impurity is surrounded instead by a LM cloud, with NFL behaviour described by the 2CK FP found in an overscreening cloud at distances $r \gg \xi_{2 C K}$.

While the phase shift concept provides an intuitive explanation for physical behaviour in models with Fermi liquid ground states, ${ }^{4}$ we point out that this is incidental. No such description in terms of phase shifts exists in the 2CK model, yet the full RG flow and universality of the problem is again recovered in real-space.

The 2CK FP is in fact the critical point of Eq. $4 .^{20,21}$ For finite channel asymmetry $\left(J_{1}-J_{2}\right) \neq 0$, a third FP enters: a Fermi liquid (FL) FP in which the impurity spin is completely screened by the more strongly-coupled channel. A new energy scale $T^{*}$ emerges, corresponding to RG flow from 2CK to FL FPs, and again shows up in the t-matrix for each channel. ${ }^{20-22}$ We find as expected that the corresponding length-scale $\xi^{*} \sim 1 / T^{*}$ characterizes real-space RG flow from a critical $2 \mathrm{CK}$ region of space to an FL region. This is shown explicitly in Fig. 4 where we compare (as in Fig. 2) the spectrum vs inverse frequency (upper panel) to the normalized densities vs distance (lower panel). Two systems are plotted, with common $K=0.01$ and $\left(J_{1}+J_{2}\right)=0.2$, but $\left(J_{1}-J_{2}\right)=0$ for the symmetric case (solid lines), while $\left(J_{1}-J_{2}\right)=10^{-7}$ for the asymmetric case (dashed lines for channel 1, dot-dashed lines for channel 2). The energy scales $T_{2 C K}$ and $T^{*}$ are indicated, as are the length scales $\xi_{2 C K}$ and $\xi^{*}$.

Characteristic asymptotic behaviour in the vicinity of $\xi_{2 C K}$ (given in the caption to Fig. 4, and shown as the dotted lines) can be extracted from Eqs. 5, 2 using the known asymptotics of the t-matrix for the symmetric $2 \mathrm{CK}$ model. Indeed, the full density crossover from $2 \mathrm{CK}$ to FL FPs for $r \gg \xi_{2 C K}$ can be calculated using the exact t-matrix announced recently in Ref. 22. Our exact result at zero-temperature and small $K$ follows as

$$
\frac{\Delta n_{i}(r)}{\Delta n_{i}^{p s}(r)} \stackrel{r \gg \xi_{2 C K}}{\sim} \pm \sqrt{\frac{2}{\pi}} \exp \left(r / \xi^{*}\right) \sqrt{r / \xi^{*}} \mathrm{~K}_{0}\left(r / \xi^{*}\right),
$$

where $K_{0}$ is the modified Bessel function of the second kind, and the $+(-)$ sign is used for the density in channel 1(2). The essentially perfect agreement between Eq. 6 (points) and the full numerical solution (lines) is shown in the lower panel of Fig. 4. Interestingly, this result indicates that the density crossover approaching the FL $\mathrm{FP}$ of the $2 \mathrm{CK}$ model is closely related to the spatial crossover in the magnetization resulting from a boundary magnetic field acting in the Ising model. ${ }^{22}$

Finally, we plot the phase diagram for the $2 \mathrm{CK}$ model in Fig. 5. Three regions of space can be identified at zero temperature, corresponding to LM, critical $2 \mathrm{CK}$ and FL FPs. The crossover length-scales $\xi_{2 C K} \sim 1 /\left(J_{1}-J_{2}\right)^{2}$ (dashed line) and $\xi_{2 C K} \sim \exp \left[1 / \max \left(\rho J_{1}, \rho J_{2}\right)\right]$ (solid line) are anticipated from the well-known variation of the energy scales $T_{2 C K}$ and $T^{*}$ in the asymmetric $2 \mathrm{CK}$ model, ${ }^{20,21}$ and agree excellently with explicit calculation (points). Even in the 'standard' case where some degree of channel asymmetry is present in the model (in which case the ground state is a Fermi liquid), the nonFermi liquid correlations at finite energies give rise to an intermediate region in space describing quantum critical behaviour.

\section{CONCLUSION}

Quantum impurity problems are generically described in terms of an RG framework: as a function of energy or distance. Specifically, static charge density oscillations away from an impurity in real-space are simply related by integral transformation to the energy-resolved scattering t-matrix - a dynamic quantity, itself related to the impurity LDOS. As such, the well-known and rich behaviour associated with RG flow between fixed points is wholly reproduced in the densities. Indeed, this is not confined to the simplest 1-dimensional geometry considered explicitly here. Although real-space behaviour does of course depend on the particular physical system under consideration, the underlying RG structure of the problem must still appear.

One implication is that the 'Kondo cloud' surrounding an impurity, typically defined as the region $r<\xi_{K}$, actually corresponds mainly to the LM FP. The screening process itself occurs on flowing to the SC FP at $r \sim \xi_{K}$.

\section{Acknowledgments}

We acknowledge funding from the Deutsche Forschungsgemeinschaft through SFB 608 and FOR 960. 
1 A. C. Hewson, The Kondo Problem to Heavy Fermions (Cambridge University Press, Cambridge, 1993).

2 D. Goldhaber-Gordon, H. Shtrikman, D. Mahalu, D. Abusch-Magder, U. Meirav, and M. A. Kastner, Nature 391, 156 (1998).

3 J. E. Gubernatis, J. E. Hirsch, and D. J. Scalapino, Phys. Rev. B 35, 8478 (1987).

${ }^{4}$ I. Affleck, L. Borda, and H. Saleur, Phys. Rev. B 77, 180404 (2008).

${ }^{5}$ G. Bergmann, Phys. Rev. B 78, 195124 (2008).

6 C. A. Büsser, G. B. Martins, L. Costa Ribeiro, E. Vernek, E. V. Anda, and E. Dagotto, Phys. Rev. B 81, 045111 (2010).

7 G. Bergmann and Y. Tao, Eur. Phys. J. B 73, 95 (2010).

8 E. S. Sørensen and I. Affleck, Phys. Rev. B 53, 9153 (1996); Phys. Rev. Lett. 94, 086601 (2005).

${ }^{9}$ L. Borda, Phys. Rev. B 75, 041307 (2007).

10 A. Holzner, I. P. McCulloch, U. Schollwöck, J. von Delft, and F. Heidrich-Meisner, Phys. Rev. B 80, 205114 (2009).

11 S. Costamagna, C. J. Gazza, M. E. Torio, and J. A. Riera, Phys. Rev. B 74, 195103 (2006).

12 T. Hand, J. Kroha, and H. Monien, Phys. Rev. Lett. 97, 136604 (2006).
13 L. Borda, M. Garst, and J. Kroha, Phys. Rev. B 79, 100408 (2009).

14 V. Barzykin and I. Affleck, Phys. Rev. B 57, 432 (1998).

15 I. Affleck, in Perspectives of Mesoscopic Physics (World Scientific, 2010), pp. 1-44.

${ }^{16}$ H. Prüser, M. Wenderoth, P. E. Dargel, A. Weismann, R. Peters, T. Pruschke, and R. G. Ulbrich, Nature Physics 7, 203 (2011).

17 See for example, J. J. Binney et al, The theory of critical phenomena: an introduction to the renormalization group (Oxford University Press, Oxford, 1992).

18 J. Friedel, Nuovo Cimento Suppl. 7, 287 (1958).

19 Low-energy behaviour is exponentially-well resolved using NRG; correspondingly, calculation of densities is most accurate at large distances. For a recent review of the NRG technique, see R. Bulla, T. A. Costi, and T. Pruschke, Rev. Mod. Phys. 80, 395 (2008).

20 P. Nozières and A. Blandin, J. Phys. II (France) 41, 193 (1980).

21 D. Cox and A. Zawadowski, Adv. Phys. 47, 599 (1998).

22 E. Sela, A. K. Mitchell, and L. Fritz, Phys. Rev. Lett. 106, 147202 (2011). 